AROUEOLOGÍA Y SOCIEDAD

№ 29, 2015: 147-166

ISSN: 0254-8062

\title{
TRAS LOS PASOS DE WENDELL C. BENNETT EN LA CUENCA DEL LAGO TITICACA
}

\author{
IN THE FOOTSTEPS OF WENDELL C. BENNETT \\ IN THE BASIN LAKE TITICACA
}

\author{
LUIS A. FLORES \\ Escuela de Antropología y Aroueología, Universidad Nacional Federico Villarreal, Perú. \\ Iflores78@gmail.com
}

\begin{abstract}
RESUMEN:
Según muchos investigadores Wendell C. Bennett fue el más importante arqueólogo extranjero de su tiempo. Su profuso trabajo le sirvió para ser considerado como uno de los fundadores de la investigación arqueológica boliviana. No obstante, su aporte ha sido muy poco considerado en el Perú, a pesar de sus múltiples excavaciones en los valles de Lambayeque y La Libertad; así como en sitios icónicos como Chavín y Huari. Esta rica información escapa de nuestro artículo, el cual se centrará solo en destacar su labor en la cuenca del lago Titicaca, teniendo en cuenta que realizó excavaciones en sitios tan importantes como Tiahuanaco y Chiripa, siendo el primero que sistematizó la información de esta región. Se propone que sus planteamientos aún siguen vigentes, muchas veces soterradamente en las interpretaciones de los arqueólogos interesados en la región altiplánica. Incluso sus formulaciones lograron ribetes panandinos con su propuesta de centros ceremoniales y Co-Tradición.
\end{abstract}

Palabras Claves: Titicaca, Tiahuanaco, Chiripa, Perú, Bolivia, arqueología andina

\section{ABSTRACT:}

According to many researchers Wendell C. Bennett was the most important archaeologist of his time abroad. His profuse work led to it being considered as one of the founders of the Bolivian archaeological research. However, their contribution has been little considered in Peru, despite its many excavations in the valleys of Lambayeque and La Libertad; as well as iconic sites like Chavin and Huari. This rich information escapes our article, which will focus only on highlighting its work in the 
Lake Titicaca, considering excavated in key places like Tiwanaku and Chiripa, the first systematized information for this region. It is proposed that their approaches are still in place, often covertly in the interpretations of archaeologists interested in the highland region. Even their formulations achieved pan-Andean trim with its proposed ceremonial centers and Co-Tradition.

KeYwords: Titicaca, Tiahuanaco, Chiripa, Peru, Bolivia, Andean archeology

\section{LA COPIOSA OBRA DE BENNETT}

Hace casi 15 años atrás, como parte de un curso de pregrado en la carrera de Arqueología de la Universidad Nacional Mayor de San Marcos, el profesor Daniel Morales ${ }^{1}$ nos hizo leer "The Peruvian Co-Tradition" de Wendell Bennett (1948) ${ }^{2}$. Esta lectura me pareció una propuesta sugestiva, en un contexto académico en la que siempre me hablaban de cómo entender los cambios culturales, pero no de cómo explicar las continuidades y entender los Andes como un todo ${ }^{3}$. Bennett al interesarse por buscar aquellas características culturales que perduraban en el tiempo y que llamó "tradición", le sumó la trascendencia temporal a la Arqueología Andina ${ }^{4}$.

Wendell Clark Bennett (Fig. 1) fue un arqueólogo norteamericano nacido en Marion, Indiana, en 1905; graduado (1927) y doctorado (1930) en la Universidad de Chicago con estudios sobre las estructuras religiosas de Polinesia. Bennett durante este tiempo había realizado estancias de investigación en Hawái y el norte de México, en todos ellos alternando tanto la arqueología como la etnografía, como dos puntales que configuraron su modo de aproximarse al pasado. Pero sería desde 1931 hasta 1938, que Bennett ${ }^{5}$ incursionaría en la cuenca del lago Titicaca, una región de altipampa de unos 800 kilómetros de extensión, por encima de los 3800 metros sobre el nivel del mar y flanqueado por cordilleras. Ha pasado más de medio siglo desde que Bennett (1934, 1936, 1948a, 1950) trabajara (y construyera) en la arqueología de esta región, en sitios tan famosos como Tiahuanaco ${ }^{6}$, Chiripa, Lucurmata y varios otros sitios. Esta investigación le daría una gran reputación académica en Estados Unidos (Kidder 1954), tanto así que hoy es considerado como uno de los fundadores de la investigación arqueológica en Bolivia (Nuevos Aportes 2007: 1; Stanish 2003: 79). Lamentablemente en Perú se conoce muy poco su aporte, a pesar que más allá del Titicaca, a él le debemos las primeras excavaciones científicas en Chavín de Huantar, donde además arrojó mucha luz sobre la cultura Recuay, en ese entonces casi desconocida. Además descubrió una manifestación, que para él era Tiahuanaco, en Wilkawain, Callejón de Huaylas, hoy uno de los ejemplos más extremos de lo que llaman Huari. En 1936 trabajó en la costa norte, en los valles de Lambayeque, Chicama, Moche y Virú. Precisamente por

1. La mención que hago del profesor Daniel Morales, en este texto, no es fortuito, es mi forma de expresar mi más sincero respeto y agradecimiento por sus enseñanzas en la Universidad de San Marcos.

2. Como el propio Bennett (1948: 1) lo ha indicado, el término "area co-tradition" fue originalmente acuñado por Ralph Linton, y fue acogido por Bennett por parecerle más satisfactorio, en un contexto en que la mayoría de investigadores ordenaba a las sociedades en "áreas culturales".

3. Esta idea de Bennett por entender la historia andina como un todo y como parte de una tradición histórica profunda, la podemos rastrear desde el capítulo publicado unos años antes en el Handbook of South American editado por Julian Steward (Bennett 1946). Aunque sus ideas germinales ya se vislumbraba desde el momento que concibió una cronología global para el Titicaca (Bennett 1936: 505).

4. Aunque Luis Lumbreras (1981: 15) criticaría posteriormente a Bennett porque -según él- la Co-tradición no habría logrado realmente darle esa dimensión temporal.

5. Bennett en dicho momento era el curador asistente de arqueología sudamericana del American Museum of Natural History de Nueva York.

6. Respetaremos el uso de Bennett del término Tiahuanaco para referirse al sitio, estilo cerámico y cultura. Dejando de lado el termino Tiwanaku que actualmente está más difundido. 
estos estudios, Gordon Willey eligió a Virú para la campaña conjunta de 1946, en la que Bennett tomó parte activa, como parte del cual excavó en el sitio de Gallinazo. Finalmente, en 1950, Bennett realizó excavaciones en varios sitios ayacuchanos, Huari, Conchopata y Acuchimachay (Bennett 1937: 249; Kidder 1954; Rouse 1954b; Tantaleán 2005). La cerámica recuperada por Bennett en Huari, aunque su estratigrafía fuera criticada, le sirvió a Dorothy Menzel (1964: 7-8) para que en parte construya su propuesta temporal y estilística para la cerámica del Horizonte Medio.

Esta amplia experiencia de la arqueología peruana le valió a Bennett para que el destacado antropólogo Julian Steward le confiera escribir el capítulo sobre la arqueología de los Andes Centrales, dentro del Volumen 2 de su famosa obra Handbook of South American Indians (Bennett 1946). Y para que el propio Luis Valcárcel (2013: 472) lo considere como un conocedor a fondo del Perú antiguo.

Revisar la obra de Bennett es una tarea pendiente de la arqueología sudamericana, aunque resulta un trabajo titánico, por lo profuso de su obra. Desde que empecé a estudiar sus textos me llamó la atención lo rápido que publicaba las monografías de cientos de hojas, solo luego de 2 o 3 años del trabajo de campo, algo realmente admirable y que hoy deberíamos calcar. Si hoy somos críticos con muchos trabajos pasados, como el del propio Bennett, es porque podemos revisar los datos que sustentaban sus hipótesis. Desafortunadamente hoy hemos dejado de publicar monografías, eso resulta ser un gran problema para la arqueología peruana, porque está permitiendo generalizaciones generosas y la aparición de literatura ficticia (Kaulicke 2000: 316).

No queda duda que Bennett contribuyó a la arqueología andina más que cualquier extranjero de su generación (Kidder 1954: 270), entonces releerlo resulta una obligación, en especial para los interesados en la cuenca del Titicaca. Desafortunadamente, el 06 de septiembre 1953, en un fatídico accidente, la arqueología en particular, la antropología y las ciencias sociales americanas en general, perdió a Bennett, cuando apenas contaba con 48 años, en plena etapa madura de su carrera (Willey 1954: 316).

En este artículo analizaré los aportes de Bennett con respecto a la arqueología de la cuenca del lago Titicaca. Empezaré por una breve explicación de cómo comprendió y construyó una historia cultural en los Andes, seguiré sus pasos por el altiplano, especialmente en los sitios Tiahuanaco y Chiripa, para finalmente hacer una reflexión sobre las implicaciones que ha tenido su trabajo en el Titicaca y en general sobre la zona andina.

\section{BenNetT y la historia CUltural De los ANDES}

Durante el tiempo de Bennett, el Historicismo Cultural era el paradigma de la arqueología. A partir de dichas ideas, los arqueólogos se preocupaban por definir los estilos culturales en cierto espacio y tiempo, en otras palabras trataron de determinar la distribución espacial y variación temporal de los tipos cerámicos, permitiéndoles definir una secuencia cronológica de dichos tipos. Siguiendo la originaria escuela germana, la arqueología tuvo como fin la construcción de una historia cultural o Kulturgeschichte. La metodología usada ampliamente por los arqueólogos fue realizar excavaciones puntuales y restringidas para obtener material, especialmente cerámico, que

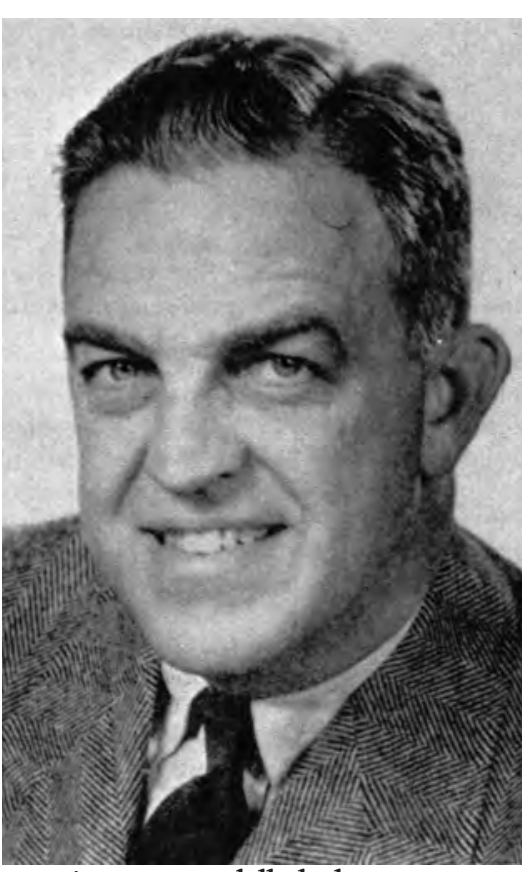

Figura 1. Wendell Clark Bennett (1905-1953) (Rouse 1954b: 265). 
pudiera ser ordenado en seriaciones estilísticas y primeros analisís estratigráficos, aunque muchos de los cuales siguieron niveles arbitrarios, sin considerar los contornos estratigráficos (Trigger 1992: 144-196).

En este ambiente teórico de principios de los años treinta del siglo XX, la arqueología andina había avanzado en un orden cronológico, construido básicamente por Max Uhle, Alfred Kroeber y sus alumnos, así como Julio C. Tello. Sin embargo, esta arqueología había llegado al límite de sus propuestas, una frontera que solo podía ser eliminada con estudios más intensos de campo (Kidder 1954: 270). Cabe recordar, que en estos tiempos se consideraba que la cultura Tiahuanaco era un estilo panandino, por la amplia distribución espacial de su estilo cerámico. Uhle (1903) lo había reportado en Pachacamac, un sitio de la costa peruana, a más de 1000 kilómetros de Tiahuanaco. Sin embargo, el sitio Tiahuanaco era estratigráficamente desconocido, el mismo Uhle había fracasado en esta tarea, por su imposibilidad de trabajar en Tiahuanaco (Kaulicke 2000: 314; Rouse 1954b: 266). Bennett era consiente de ese contexto y la posición clave que resultaba entender la secuencia estratigráfica y estilística de Tiahuanaco, por ello eligió excavar en Bolivia.

\section{Sus Pasos Por el Altiplano: los años 30 del siglo XX}

El turbulento escenario político de Bolivia era ya insostenible para 1920. Un país que había crecido de manera desigual, gracias a la explotación de un mineral, el estaño, pero apoyándose en la fuerza laboral de los más débiles e ignorados, la gente campesina y minera. Todo esto desembocó en detrimento de la clase política, una nación que apenas empezaba a construirse, sin liderazgo para entender las contradicciones sociales. Tal vez la insubordinación más icónica fue la llamada rebelión de Chayanta, Potosí, durante 1927, donde los campesinos se levantaron en contra de los hacendados. Junto a este desequilibrio político, Bolivia afrontó por esos años la crisis económica mundial de 1929, que trajo consigo la baja del precio del estaño en 1931. A toda esta crisis social y económica se sumó la Guerra del Chaco entre 1932 y 1935 (Antezana 1979).

En medio de esta zozobra e incertidumbre política y económica que vivía Bolivia, llega al altiplano Wendell Bennett, en compañía de John G. Phillips, para iniciar una serie de investigaciones arqueológicas en las tierras altas, entre marzo a setiembre de 1932. La primera exploración partió desde La Paz, siguiendo el lado oriental del lago Titicaca hasta Escoma, y de allí al interior hacia Mocomoco. En este viaje describió, fotografió y mapeó más de cuarenta pueblos y fortificaciones identificadas como incas. El segundo viaje fue a Cochabamba y la región circundante; aquí exploró diferentes montículos. Durante los meses de junio y julio del mismo año, el gobierno boliviano finalmente autorizó la excavación de diez pozos de prueba en Tiahuanaco ${ }^{7}$. Este trabajo reveló una estratigrafía y secuencia cerámica importante para entender el problema cronológico y cultural que buscaba resolver Bennett (1934:361).

Después de Tiahuanaco, Bennett acabó su visita en las islas del Titicaca, Coati y la península de Copacabana, donde identificaría posibles afiliaciones Tiahuanaco e Inca. Finalmente, dejaría el altiplano para visitar Cusco y la costa peruana (Bennett 1934: 361-2).

Un año después, en diciembre de 1933, Bennett regresó a Bolivia para continuar con sus investigaciones en el altiplano, como parte de una expedición del American Museum of Natural History financiado por Voss Fund. Durante los primeros cinco meses también participó Junius Bird como asistente. Pero el trabajo se vio retrasado por la Guerra del Chaco, lo que demoró los permisos para el ingreso al país

7. Ponce Sanginés (1999: 71) señala que Wendell Bennett recibió dicha autorización gracias a las gestiones del diplomático estadounidense Edward F. Feeley. 
y para las excavaciones. Durante esta segunda expedición llegó a excavar alrededor de la ciudad de Cochabamba. Los cateos fueron realizados en Tiquipaya, un montículo clasificado como Tiahuanaco Decadente. Realizó también excavaciones en las localidades de Colcapirhua y Arani, que resultó en el descubrimiento de muchas tumbas del tipo Tiahuanaco. Además excavó en Llogheta, un montículo Tiahuanaco dentro de los límites del municipio de La Paz. Y practicó excavaciones en varios sitios incas en la isla del Titicaca, empero resultaron infértiles para determinar si existía una ocupación previa (Bennett 1936: 331-2).

Pero tal vez la principal excavación que llevó a cabo durante esta temporada, fueron las cinco semanas de trabajo en el montículo artificial Chiripa, en el borde del lago Titicaca, península de Taraco, cuyos resultados ampliaremos más adelante. Finalmente, Bennett dedicó seis semanas de excavación en Lucurmata, al borde del lago Titicaca, otro sitio tipo de Tiahuanaco Clásico. Bennett (1936: 332) se quedaría en el altiplano hasta septiembre de 1934.

\section{BennetT en Tiahuanaco}

A través de la resolución suprema del 19 de mayo de 1932, el gobierno boliviano autorizó a Wendell Bennett realizar investigaciones arqueológicas en Tiahuanaco, permaneciendo en el sitio desde el 15 de junio hasta el 10 de julio de 1932 (Ponce 1999: 70). Bennet (1934) excavó 10 pozos distribuidos tanto en el centro del sitio como en las zonas periféricas. Gracias a este trabajo, basado principalmente en el estilo de la cerámica (de los 14,500 tiestos cerámicos recuperados), su posición en los pozos excavados, los períodos de construcción y la litoescultura, Bennett llegó a establecer la primera secuencia para Tiahuanaco, siguiendo un orden evolutivo (Kidder 1954: 270): Tiahuanaco Temprano o pre-Tiahuanaco, Tiahuanaco Clásico, Tiahuanaco Decadente, Post-Tiahuanaco e Inca.

De los pozos excavados, dos se efectuaron sobre estructuras arquitectónicamente importantes. El pozo II fue planteado en el Kalasasaya y el pozo VII en el Templo Semisubterraneo o Templo Este (como lo llamó Bennett), donde se encontraron cuatro litoesculturas: una estatua monolítica grande (monolito Bennett), otra pequeña (Monolito Barbudo) y dos estelas más de piedra (Bennett 1934) (Fig. 2).

Sin embargo, los pozos V y VIII fueron los que dieron mejores resultados estratigráficos para respaldar la secuencia. En los niveles 6 al 9 del pozo $\mathrm{V}$ y en los niveles 4 al 6 del pozo VIII se encontró cerámica Tiahuanaco Temprano. En los niveles 3 al 5 del pozo $\mathrm{V}$ y en el nivel 3 del pozo VIII se encontró Tiahuanaco Clásico. En los niveles 2 al 3 del pozo V y en el nivel 1 del pozo VIII se encontró Tiahuanaco Decadente (Bennett 1934).

Lo que Bennett llamó Tiahuanaco Temprano, en realidad era una distribución de cerámica bruñida de varios colores, como negro, blanco, rojo, naranja, marrón y colores brillantes aplicados directamente a una arcilla amarillenta sin engobe. Los diseños más característicos de esta cerámica fueron zigzag con colores alternados, o zoomorfas con animales peculiares, pintados de colores sobre un fondo negro. Las áreas de diseño fueron limitadas. Parte de la decoración se hizo rellenando líneas incisas con colores probablemente perteneciente a este grupo. Propio de estos niveles también fue el hallazgo de pequeños botones de arcilla y hachas de piedra en forma de T (Bennett 1934). La distribución de este estilo se limitaba a tres sitios (Tiahuanaco, Chiripa y Kea Kolo Chico en la isla del Titicaca) y a sólo dos sub-áreas (Tiahuanaco y Copacabana). Sin embargo, Bennett se daría cuenta que estilísticamente Tiahuanaco Temprano no tenía relación con Tiahuanaco Clásico. Por ello sostuvo que el propio nombre de dicho periodo estaba mal definido. Posteriormente, Bennett $(1948,1950$ : 91) descubre que estratigráficamente el estilo Tiahuanaco Temprano se ubicaba encima de la cerámica Chiripa y por debajo de Tiahuanaco Clásico. 
Para el periodo Tiahuanaco Clásico, Bennett sostiene que existe una buena evidencia de asociación de arquitectura (templos y plataformas con patios hundidos, portadas monolíticas y muros con pilares verticales con bloques pétreos en el centro), estatuas y sus tipos de cerámica policroma. La cerámica típica es de tres o más colores, como negro, blanco, amarillo, y, a veces gris y marrón sobre una base roja, así como cerámica roja pulida. El esquema lineal en negro es distintivo. La forma más característica es el kero, aunque también son frecuentes los tazones de lados expandidos para libación. Los diseños más repetidos son perfiles de pumas, cóndores y humanos, así como unidades geométricas (Bennett 1934, 1950).

Por su parte, lo que llamó Tiahuanaco Decadente fue planteado como una degeneración del Clásico, aunque combinado con algunos elementos nuevos. Las formas kero e incensario todavía se encontraban en el registro, aunque la forma más representativa es una taza de fondo plano. La típica combinación de colores son blanco y negro aplicado sobre un engobe naranja. Hay un mayor énfasis en el diseño geométrico, la doble S y el zigzag. El diseño zoomorfo representa un desglose de las formas clásicas de cóndor, puma y figura humana, en burdas imitaciones o diseños basados en un ala del cóndor, una cabeza de puma, ojos de un ser humano, etc. En general se produce una decadencia frecuente en el acabado, la aplicación, la textura y el pulido. Finalmente, material Inca se superpone a Tiahuanaco, pero sin implicaciones de conexión histórica. El Post-Tiahuanaco, llamando entonces "Chullpas" resultaba común de Bolivia (Bennett 1934).

Como he descrito, fueron importantes sus hallazgos y los primeros intentos de relaciones estratigráficas con implicaciones culturales. Sin embargo, el trabajo de Bennett no estuvo, ni está exento de críticas, especialmente por su método de excavación arbitraria, que en muchos casos trajo consigo la mezcla de materiales (Menzel 1964; Machicado 2009). No obstante, este es un error que él mismo lo reconoció, pues dichos niveles arbitrarios en muchos casos no permitió que coincidiera con las unidades estratigráficas (Bennett 1934:369, 447). Ello respondería el por qué en ciertos niveles se descubría cerámica Tiahuanaco Clásico mezclada con Tiahuanaco Decadente, siendo tal vez el mejor ejemplo lo sucedido en el pozo IV. Esta composición ha hecho que algunos autores sostengan que es difícil distinguir estos tipos cerámicos como dos periodos distintos (Janusek 2003: 82, Machicado 2009: 52). Además, cabe anotar que los pozos V y VIII, sobre cuya estratigrafía se basa la secuencia de Bennett, se ubicaron dentro del sitio; pero en espacios no edificados, al este y oeste de Akapana. Por tanto quedó un vacío por entender la historia constructiva del sitio.

A pesar de las detracciones recientes, no hay que olvidar que fue gracias a la investigación de Bennett (1934) que se estableció la primera secuencia para Tiahuanaco, y en general para la cuenca del Titicaca. Su planteamiento fue un viraje sustancial de los enunciados dominantes de la época, donde sobresalía la figura de Arthur Posnansky (Ponce 1999: 71). Es así que el trabajo de Bennett sentó las bases para todos los estudios posteriores sobre la idea de propagación de la influencia de Tiahuanaco sobre los Andes Centro-Sur y Centrales, un problema de investigación en el que Bennett se interesó durante el resto de su vida, lo que lo llevó en 1950 a excavar en Wari y otros sitios andinos (Bennett 1934: 476; Kidder 1954: 270).

\section{BennetT en ChIRIPA}

En 1934, Bennett regresó a Bolivia, ampliando el conocimiento de Tiahuanaco, tanto de las tierras bajas, como de la cuenca del Titicaca. Estas labores consistieron en excavaciones de sitios hasta ese entonces desconocido para la civilización Tiahuanaco. Destaca sus trabajos en Lucurmata y una distribución más amplia de materiales de Tiahuanaco Clásico y Decadente. También observó algunos sitios incas, examinando dichas evidencias en las islas de Quehuaya, Paco, de Cumaná y en Pariti, aunque no se distrajo tanto en ellos (Bennett 1936). 


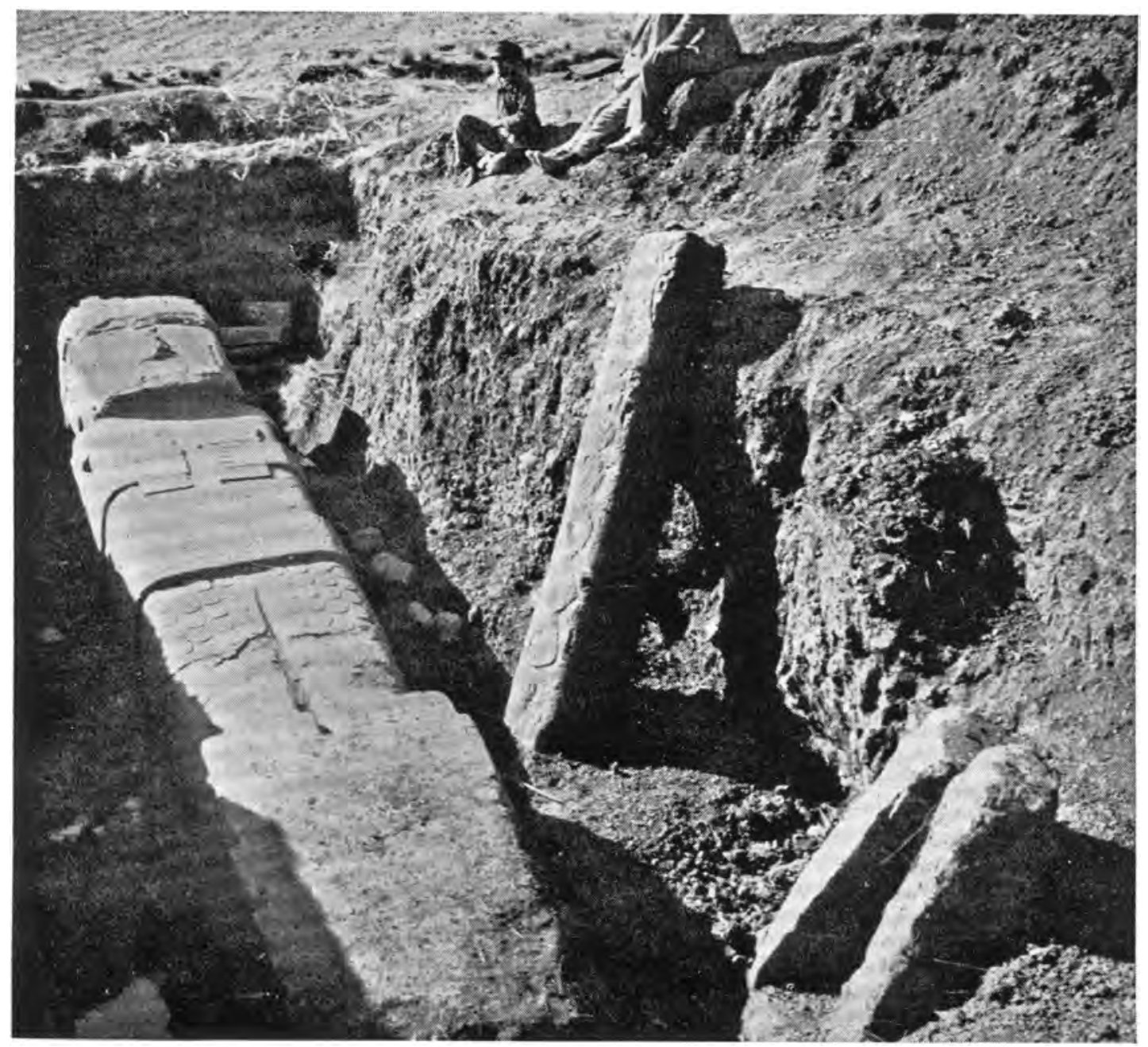

Figura 2. El descubrimiento de los monolitos, que más tarde se conocerán como monolito Bennett y monolito Barbado. Excavaciones en el pozo VII de Tiahuanaco (Bennett 1934: Fig.20).

El resultado más importante de esta expedición fue el descubrimiento de la cultura que llamó Chiripa. Bennett (1936: 332) en este sitio realizó una trinchera, de aproximadamente treinta metros de largo, que se ubicó desde el borde hasta el centro del montículo, que en al menos tres lugares llegó a profundizar hasta los cinco metros. Además hizo una serie de cateos restringidos al interior y exterior del montículo. Se estima que fueron diez pozos en el montículo y al menos seis trincheras fuera del mismo, en el campo, con la finalidad de ubicar tumbas y estratos anteriores al montículo (Bennett 1936: 413-5). Desafortunadamente sobre dichas excavaciones exteriores informó muy poco (Machicado 2009: 74-5), en parte porque encontró pocas evidencias.

En general, gracias a la sección estratigráfica del perfil oeste del sitio pudo definir una secuencia de cuatro agrupaciones estratigráficas, al menos notorio en el extremo norte de dicho perfil. El más antiguo e inferior es una ocupación sin arquitectura corporativa que llamó el Pre-montículo, que se corresponde con las evidencias descubiertas al exterior norte, por tanto estos niveles se extienden 
más allá del montículo, donde destacan evidencias de muros de piedra, de tal vez viviendas informales, niveles de ceniza, restos de peces, animales y entierros quizá humanos. Encima de estos niveles, varios niveles de depósitos, que configuraron las primeras evidencias de arquitectura monumental, que soportó un circulo de casas, en un diámetro en conjunto de 32 metros; al parecer esta ocupación fue destruido y quemado, así fue interpretado por Bennett debido a la presencia de abundante ceniza y quemas sobre el piso, dejando un montículo bajo, con una ligera depresión en el centro. La siguiente fase estuvo representada por estratos que cubrieron los viejos recintos, construyendo un templo encima, que representó una ampliación de todo el montículo, donde destaca un templo semi-subterráneo de piedra construido en el centro de la ocupación; además se colocaron entierros cortando las viejas paredes de lo que Bennett llamó Casa 2. Por último, una fase post-templo, en el que el templo en sí fue parcialmente rellenado con escombros. Estas agrupaciones estratigráficas pudieron ser reunidas en dos grandes periodos, distinguidos además por culturas distintas; desafortunadamente solo se pudo distinguir el periodo superior que corresponde desde la construcción del templo, que fue identificado originalmente, como veremos más delante erróneamente, como del periodo decadente de Tiahuanaco (Bennett 1936: 430-431 y Fig.24).

Precisamente, en cuanto a su materialidad mueble, este último periodo de Chiripa fue caracterizada por un tipo de cerámica de ollas, vasos y cuencos de color marrón claro, generalmente sin asas y de color amarillo sobre rojo o negro; con diseños simples de triángulos aplicados en la parte exterior de los vasos y otros lineales en el borde interior, también se usan líneas incisas y la técnica de modelado de relieve de animales. Aunque originalmente Bennett (1936:502) consideró esta cerámica Chiripa como post-Clásico y pre-Tiahuanaco Decadente, tuvo claro desde un inicio que no se trataba de un sub-estilo Tiahuanaco. Ulteriormente, gracias a una revisión cuidadosa de sus notas de campo y colecciones de Tiahuanaco, Chiripa y Pariti, y los nuevos datos de M. Tschopik y Kidder II en Pukara en el norte del Titicaca, Bennnett se daría cuenta que su interpretación estratigráfica estuvo equivocada en ciertos puntos. Precisamente con el propio Kidder II, en 1947, reevaluaron la cronología y las relaciones culturales de varias colecciones del altiplano, pero especialmente de Chiripa y Pucara (Franquemont 1986: 1). Por ello, casi quince años después de sus trabajos en el Altiplano, Bennett se retractó de su planteamiento original, considerando a Chiripa en una posición estratigráficamente anterior a Tiahuanaco Temprano (Bennett 1948a, 1950; Kidder 1954: 270).

Bennett sostuvo finalmente que Chiripa fue la cultura y periodo más antiguo de la parte sur de la cuenca, distinguible básicamente por la distribución de una cerámica amarillo sobre rojo, aunque él la encontró limitada a pocos sitios (Tiahuanaco, Lloguera, Pajchiri, Pariti, Copacabana y obviamente Chiripa) y en solo dos sub-áreas (Copacabana y Tiahuanaco). Según él esta cultura se distinguió por el patrón monticular (Fig. 3) que soportaba un grupo circular de quizás catorce "casas" rectangulares, formados por muros dobles de piedra con entierros debajo de las estructuras y al parecer con techos de paja (Bennett 1934; 1948a: 90; 1950: 90-91). Lamentablemente a Bennett le faltó informar mejor sobre el sistema constructivo de los edificios y sus relaciones estratigráficas (Machicado 2009: 83)

\section{BENNETT Y SU IDEA DE UNIDAD Y DIVERSIDAD CULTURAL. LA CUENCA DEL TITICACA VISTA EN CONJUNTO}

Bennett (1950: 89) consideró que el territorio más adecuado para la morada de las sociedades antiguas en la cuenca del Titicaca se limitaba en gran parte a las inmediaciones del Titicaca, dentro de un área de 31 mil kilómetros cuadrados, incluyendo el lago. 


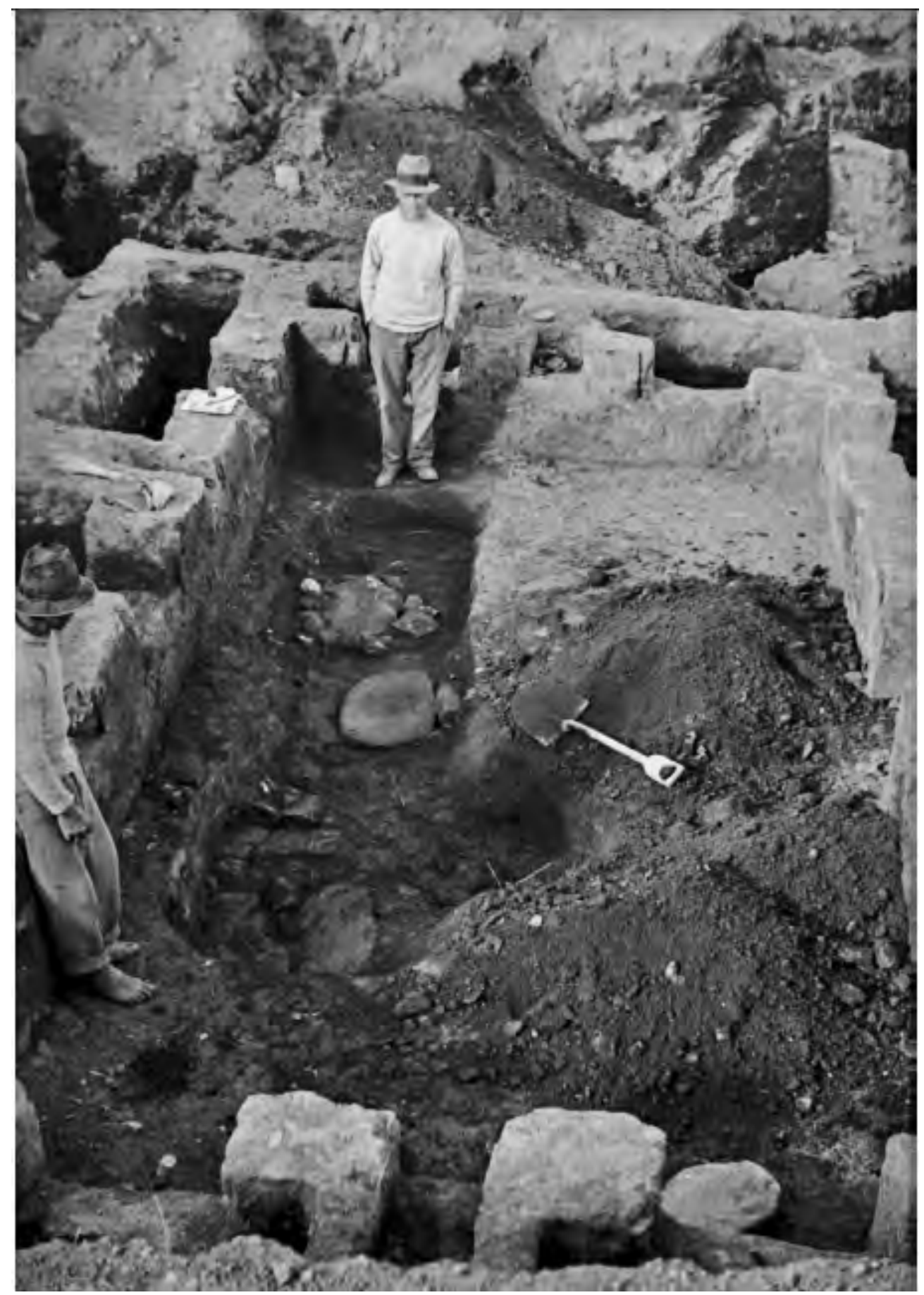

Figura 3. Wendell Bennett durante las excavaciones debajo del piso de "House 2", sobre el Montículo de Chiripa (Machicado 2009: Fig.4.10, de los Archivos del American Museum of Natural History, NY). 
1. Tiahuanaco, que incluye la región al sur del lago.

2. Copacabana, que se refiere a la península del mismo nombre,

3. Las islas de la sección boliviana del lago y las islas del Titicaca y Coati.

4. Achacache, la costa sureste del lago hasta la frontera con Perú.

5. Moho en el noreste,

6. Taraco, al noroeste de Puno,

7. Juli, la parte sudoeste del lago orilla.

En estas subzonas se distribuían las unidades culturales, que para ser objetivos, básicamente Bennett se refería a la distribución espacial de un estilo de cerámica y secundariamente de tipos arquitectónicos y litoescultura. Según esta idea, pocos fueron los periodos que se logró una unidad cultural de toda la región (Fig. 4 y Fig. 5) 8 .

Estas siete subzonas fueron agrupadas por Bennett (1950) en dos grandes zonas:

1. La Cuenca Sur del Titicaca: formado por las subzonas Copacabana, Tiahuanaco y Achacache. Y cuyos sitios fueron distribuidos en un orden cronológico, de abajo hacia arriba, en los periodos Chiripa, Tiahuanaco Temprano, Tiahuanaco Decadente, Khonkho e Inca.

2. La cuenca Norte del Titicaca: formado por las subzonas Moho, Taraco, Puno y Juli. Y cuyos sitios fueron distribuidos en un orden cronológico, de abajo hacia arriba, los periodos Pukara, Tiahuanaco Decadente, Allita Amaya-Collao-Sillustani y Taraco-Inca-Chucuito.

Esta revisión de la información del Titicaca le sirvió a Bennett (1950) para realizar una discusión general de la cuenca en su conjunto. Sostuvo que la evidencia arqueológica apuntaba consistentemente a la falta de unidad global durante gran parte del tiempo. Además, sustentó que las áreas más favorables para el desarrollo cultural se encontraban en los extremos norte y sur del lago.

En un primer periodo, el norte fue dominado por la cultura Pukara, mientras el sur por Chiripa y Tiahuanaco Temprano. Por tanto, según esta propuesta de Bennett (1950), parecería que estas dos áreas nunca se unieron, salvo en momentos excepcionales.

La primera sugerencia de la existencia de una unidad cultural en la cuenca fue durante el período Tiahuanaco Decadente. Sin embargo, dicha cerámica en el norte de la cuenca eran muy rara de encontrar. Más tardíamente, se daría un paisaje político atomizado, con la distribución de los estilos Khonkho en el sur de la cuenca y Sillustani, Collao Negro sobre Rojo y Allita Amaya en la parte sur. Para Bennett (1950) el único periodo de consolidación regional claro sucedió durante la época incaica. Una vez más la concentración de los sitios se localizaba en los extremos norte y sur de la cuenca. En el norte, los estilos locales Chucuito fue reportado en la sub-zona de Puno, mientras Taraco policromada en la subzona de Taraco (Bennett 1950).

Este panorama dividido de la organización espacial y político de la cuenca del Titicaca se vio reflejado en el momento de la conquista Inca. Los nativos sostenían que la región fue ocupada por cuatro grupos principales, cada uno con diferentes dialectos, y rara vez unidos. El grupo Colla que ocupó la sub-área Taraco, el grupo Lupaca ocupó las sub-áreas de Puno y Juli, el grupo Pacasa ocupó la sub-área de Tiahuanaco y el grupo Omasuyu en las subáreas Achacache y Moho. Estos grupos corresponden generalmente a las divisiones que han usado los arqueólogos (Bennett 1950).

8. Como ya he señalado antes, esta idea de unidad y separación cultural ya lo venía gestando Bennett desde el momento que concibió una cronología global para el Titicaca (Bennett 1936: 505). 


\section{LA HUELLA DE BENNETT EN LA ARQUEOLOGÍA DE LA CUENCA DEL TITICACA Y SUS IMPLICANCIAS EN LA ARQUEOLOGÍA ANDINA}

La huella de Bennett aún está presente en la arqueología de la cuenca del lago Titicaca. Sus propuestas han quedado marcadas como pisada sobre un cemento fresco, en los albores de la disciplina. Esa huella lo identificamos aun en la secuencia cultural y en las propuestas de desarrollo histórico para Tiahuanaco.

Como bien lo han señalado otros autores (Albarracin-Jordan 2007: 28; Lumbreras 1982: 6; Tantaleán 2005: 156), la secuencia histórica-cultural propuesta por Bennett para la cuenca del Titicaca lo han ratificado y replicado varios otros arqueólogos como Alfred Kidder II y Carlos Ponce Sanginés. Y si bien recientemente hay nuevas propuestas de ordenamiento temporal para Tiahuanaco (Burkholder 2001; Janusek 2003), no hay duda que la secuencia original de Bennett aún está presente soterradamente.

Además, le debemos a Bennett la idea ilusoria de solo dos focos de desarrollo en la cuenca, uno al norte con Pukara y otro al sur con Tiahuanaco (Fig. 5). Si bien esta propuesta ha sido superada, por una que postula un panorama político atomizado para el periodo Formativo, aun ella sale a flote entre los investigadores. Por ejemplo, Charles Stanish $(2001: 199,208)$, tal vez uno de los que mejor conoce este periodo, es claro en señalar un escenario político conformado con numerosos sistemas políticos compitiendo en la región, aunque gran parte de su texto se centra en la discusión de Pukara y Tiahuanaco por considerarlos como los dos principales sistemas políticos. Entonces, en cierta medida, la idea de Bennett aún persiste.

A nivel macro, la idea de Bennett sobre el sitio Tiahuanaco marcó en gran medida los conceptos que aun usamos los andinistas para referirnos a las primeras agrupaciones poblacionales. Una de estas concepciones es la idea de centro ceremonial, es decir un complejo de edificios usados eventualmente para actos rituales, pero sin ocupación permanente, tal como lo definió más tarde John Rowe (1963). Obviamente cuando Rowe planteó esta concepción para los Andes, ya se conocían las ideas de Ephraim Squier (1877: 300) sobre Tiwanaku y del propio Wendell Bennett (1934: 480) quien sostuvo que: “...Tiwanaku es definitivamente un sitio ceremonial, compuesta por una aglomeración de templos".

\begin{tabular}{|c|c|c|c|c|c|c|c|c|}
\hline \multicolumn{4}{|c|}{ Cuenca Sur del Titicaca } & \multicolumn{5}{|c|}{ Cuenca Norte del Titicaca } \\
\hline PERIODOS & $\begin{array}{l}\frac{\pi}{2} \\
\text { d } \\
\text { d } \\
0 \\
0 \\
0\end{array}$ & 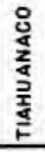 & 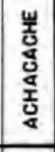 & 욷 & $\begin{array}{l}8 \\
\vdots \\
\alpha \\
\alpha \\
\alpha\end{array}$ & 足 & ذَ & PERIODOS \\
\hline INCA & 9 & 8 & $2+$ & + & $\begin{array}{l}2+ \\
8+ \\
3 \\
\end{array}$ & $\begin{array}{c}5+ \\
17+ \\
2\end{array}$ & $\frac{1}{2}$ & $\begin{array}{c}\text { Chucuito } \\
\text { INCA } \\
\text { Taraco }\end{array}$ \\
\hline KHONKHO & 2 & 4 & $20+$ & 5 & $\begin{array}{c}2+ \\
9\end{array}$ & $\begin{array}{c}3+ \\
2 \\
1\end{array}$ & + & $\begin{array}{l}\text { Sillustani } \\
\text { COLLAO } \\
\text { Allita Amayo }\end{array}$ \\
\hline $\begin{array}{l}\text { TIAHUANACO } \\
\text { DECADENTE }\end{array}$ & 4 & 7 & 2 & & 1 & 4 & I & $\begin{array}{l}\text { TIAHUANACO } \\
\text { DECADENTE }\end{array}$ \\
\hline $\begin{array}{l}\text { TIAHUANACO } \\
\text { CLASICO }\end{array}$ & 6 & 6 & & & \multirow{3}{*}{4} & \multirow{3}{*}{1} & \multirow{3}{*}{2} & \multirow{3}{*}{ PUCARA } \\
\hline $\begin{array}{l}\text { TIAHUANACO } \\
\text { TEMPRANO }\end{array}$ & 1 & 2 & & & & & & \\
\hline CHIRIPA & 3 & 3 & & & & & & \\
\hline
\end{tabular}

Figura 4. División cronológica de los sitios según las zonas de la cuenca del Titicaca propuesta de Wendell Bennett (1950: fig.35). 
Finalmente, a Bennett (1948) le debemos la idea de Co-tradición, un intento por agrupar, de manera interconectada, las tradiciones culturales a lo largo de un tiempo y espacio. Y a diferencia de la clasificación del área cultural, las culturas incluidas en un área de co-tradición fueron tratadas como totalidades. Por lo tanto cada uno tenía su propia historia, sus propias tradiciones persistentes. Esta visión de Bennett buscaba que los arqueólogos no solo investiguemos los cambios, sino aquellos rasgos culturales que permanecen en el tiempo y que llegan a definir una tradición. Esta propuesta es sumamente interesante. Desafortunadamente, luego de Bennett, la co-tradicción ha sido poco desarrollada (Rouse 1954a). Un nueva lectura de esta idea debería inspirar nuevos cambios en la forma como construimos y periodizamos nuestra historia andina.

Al final de este texto o mejor dicho, hasta que las fuerzas nos dieron para seguir los incansables pasos de Bennett por la puna, como conclusión queda que su rastro e implicancias en la constitución de la arqueología de la cuenca del lago Titicaca resultó fundacional e inspiradora. Ya dependerá de las nuevas generaciones aprender a caminar como él, con pasos rápidos.

Figura $5(1,2,3,4,5,6)$

Distribución espacial de los principales sitios por periodos y zonas culturales según la propuesta de Wendell Bennett para la cuenca del Titicaca (1950).

\section{1.- Periodo PUCARA/CHIRIPA}

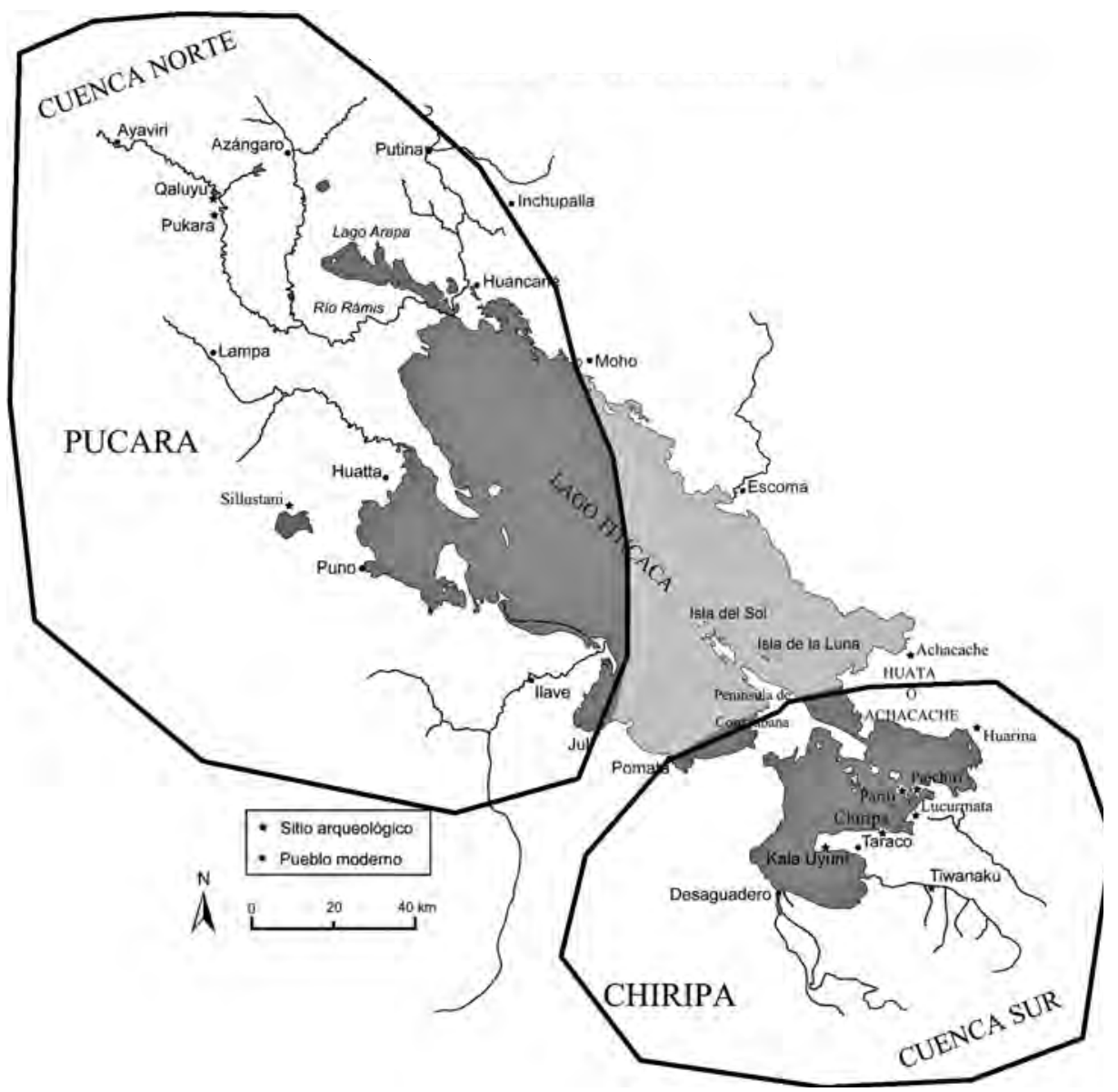




\section{2.- Periodo PUCARA/TIAHUANACO TEMPRANO}

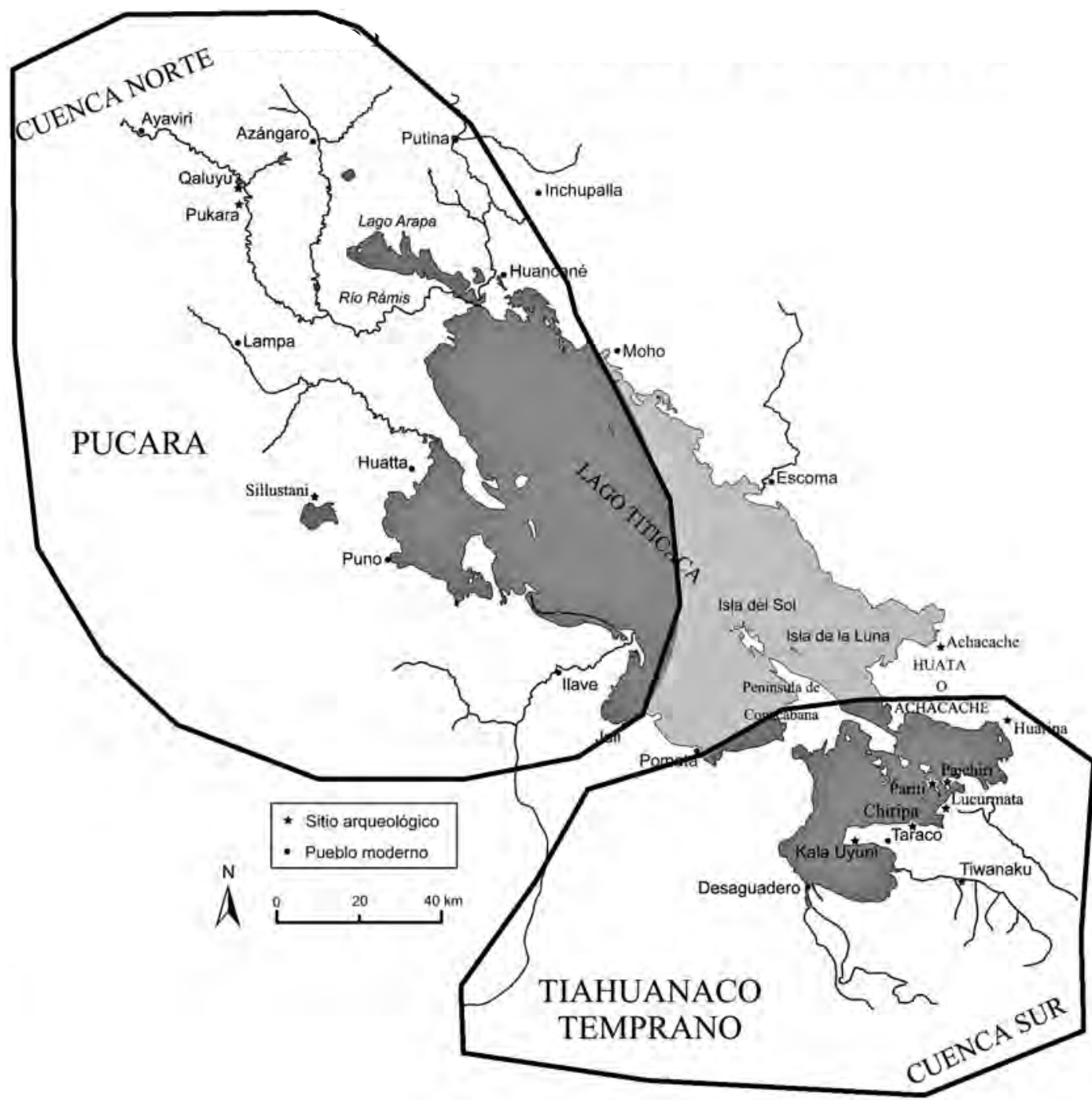




\section{3.- Periodo PUCARA/TIAHUANACO CLASICO}

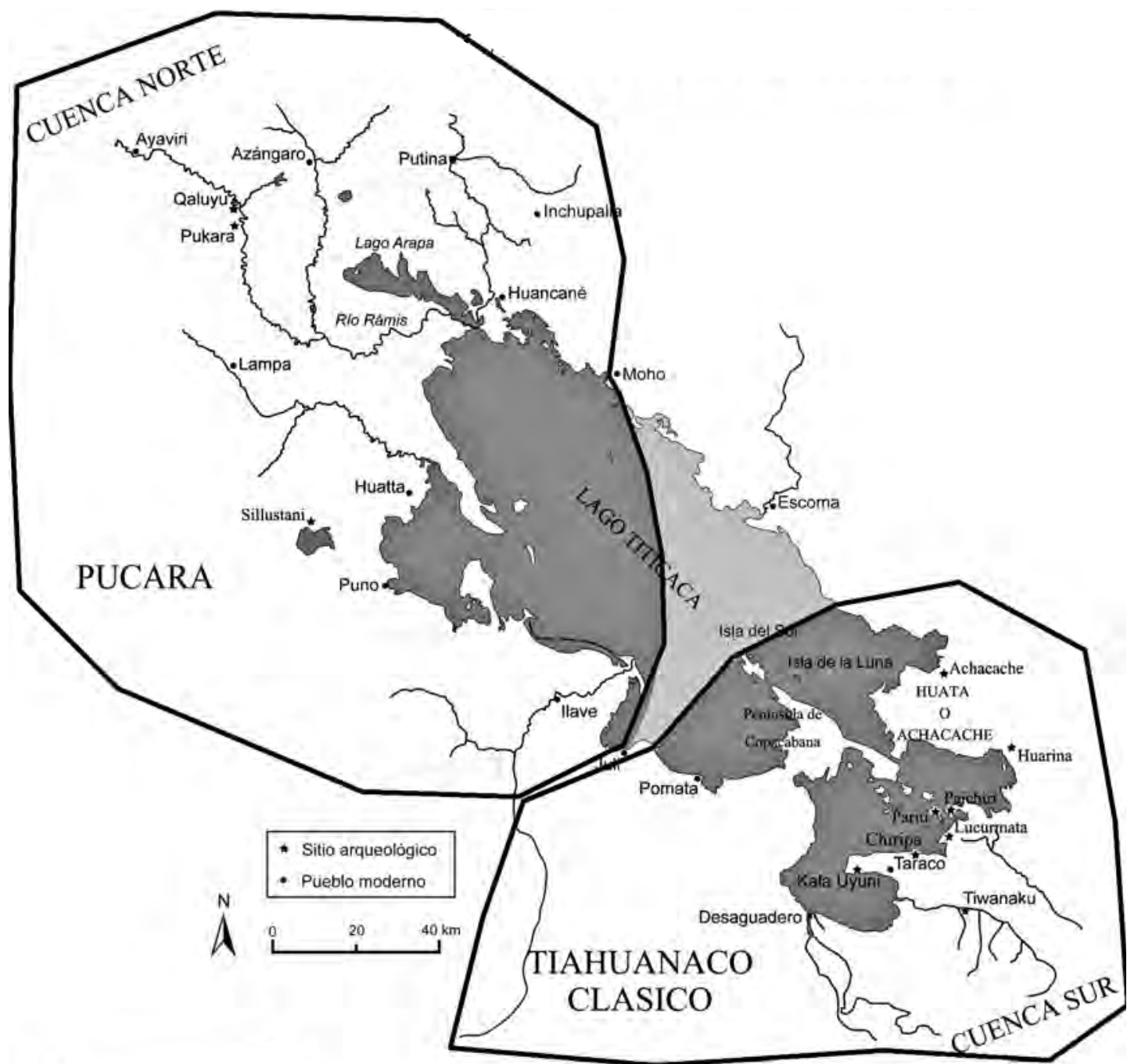




\section{4.- Periodo TIAHUANACo DECADENTE}

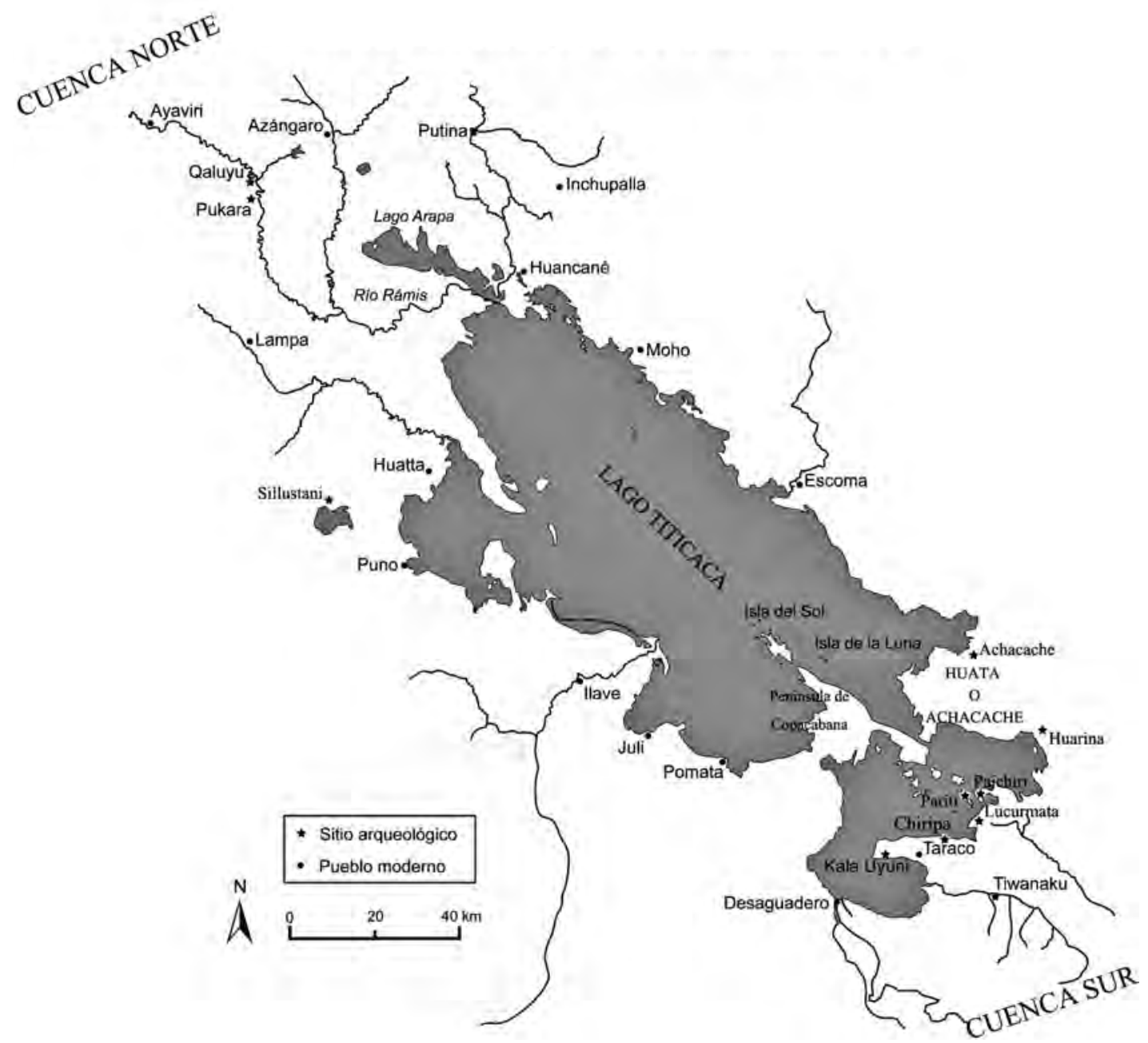




\section{5.- Periodo SILLUSTANI/KHONKHO}

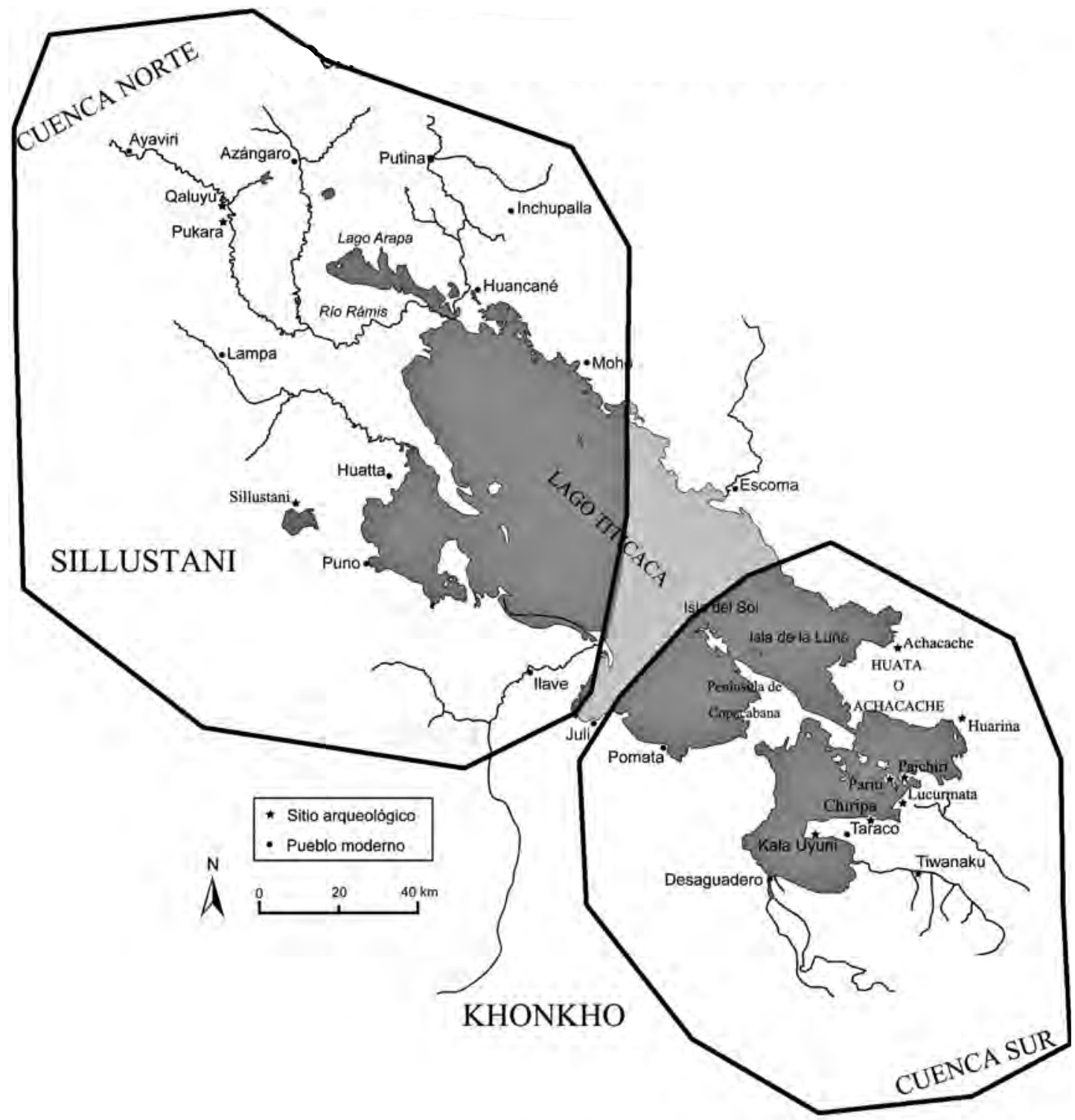




\section{6.- Periodo INCA}

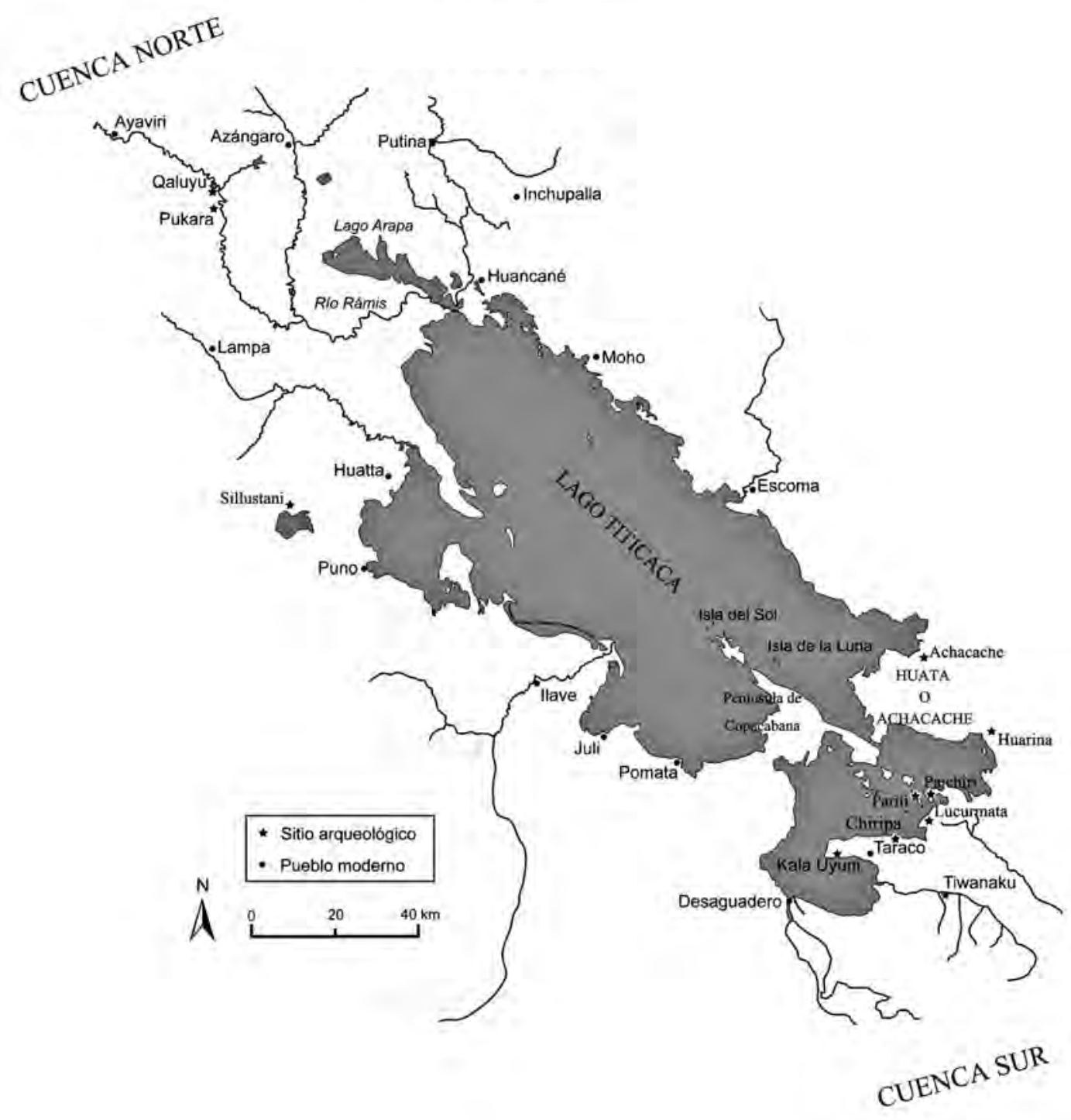




\section{BiBLIOGRAFÍA 9}

ALBARRACIN-JORDAN, Juan

2007 La formación del Estado prehispánico en los Andes. Origen y desarrollo de la sociedad segmentada indígena. Fundación Bartolomé de las Casas.

ANTEZANA VILLAGRÁN, Jorge

1979 La Guerra del Chaco. Vol. 2. Editorial Calama.

BENNETT, Wendell C.

1934 Excavations at Tiahuanaco. Anthropological papers of The American Museum of Natural History, NY. Volume XXXIV, Part III.

BENNETT, Wendell C.

1936 Excavations in Bolivia. Anthropological papers of The American Museum of Natural History, NY. Volume XXXV, Part IV.

BENNETT, Wendell C.

1937 Archaeology Archaeological Work in South America, 1934 to 1936. American Antiquity, 2 (4): 248-259.

BENNETT, Wendell C.

1946 The Archeology of the Central Andes. En Handbook of South American Indians, editado por Julian Steward. BAE-B, No. 143, Vol.2: 61-147.

BENNETT, Wendell C.

1948a A Revised Sequence for the South Titicaca Basin. Memoirs of the Society for American Archaeology, 4: 90-92. Menasha.

BENNETT, Wendell C.

1948b The Peruvian Co-Tradition. En Memoirs of the Society for American Archaeology, No. 4, A Reappraisal of Peruvian Archaeology, pp. x, 1-7.

BENNETT, Wendell C.

1950 Cultural Unity and Disunity in the Titicaca Basin. American Antiquity, 16 (2): 89-98.

BENNETT, Wendell C.

1953 Area Archeology. American Anthropologist, 55(1): 5-16.

BURKHOLDER, Jo Ellen

2001 “La cerámica de Tiwanaku: ¿qué indica su variabilidad?” Boletín de Arqueología PUCP, 5: 217249.

FRANQUEMONT, Edward M.

1986 The ancient pottery from Pucara, Peru. Ñawpa Pacha, 24: 1-30. (1954b: 268-270). 
JANUSEK, John

2003 Vessels, Time and Society: Towards a ceramic chronology in the Tiwanaku Heartland. En Tiwanaku and its Hinterland (Alan Kolata, ed.). Pp. 30-91. Smithsonian Institution Press, Washington D.C.

\section{KAULICKE, Peter}

2000 La Sombra de Pachacamac: Huari en la Costa Central. En: Boletín de arqueología de la PUCP. Nº: 313-358. Pontificia Universidad Católica del Perú. Lima.

KIDDER II, Alfred

1954 Wendell Clark Bennett 1905-1953. American Anthropologist, 56(2): 269-273.

LUMBRERAS, Luis G.

1981 Arqueología de la América Andina. Ed. Milla Batres, Perú.

MACHICADO, Eduardo

2009 Las tumbas de la península de Taraco: trayectorias de cambio en prácticas funerarias durante la transición entre el Formativo Medio y el Formativo Tardío. Tesis de Licenciatura, Universidad Mayor de San Andrés, La Paz, Bolivia.

MENZEL, Dorothy

1964 Style and Time in the Middle Horizon. Ñawpa Pacha 2: 1-106. Berkeley, California.

NUEVOS APORTES

2007 Editorial. El rol de las universidades en la investigación arqueológica en Bolivia. Nuevos Aportes 4: 1-3. Bolivia.

PONCE SANGUINÉS, Carlos

1999 Arthur Posnansky y su obsesión milenaria. Biografía intelectual de un pionero. Producciones Cima. La Paz, Bolivia.

ROUSE, Irving

1954a On the use of the Concept of Area Co-Tradition. American Antiquity, 19 (3): 221-225.

ROUSE, Irving

1954b Necrology: Wendell C. Bennett 1905-1953. American Antiquity, 19 (3): 265-270.

ROWE, John H.

1963 Urban Settlements in Ancient Peru. Nawpa Pacha 1: 1-27.

SQUIER, Ephraim G.

1877 Peru; incidents of travel and exploration in the land of the Incas. New York: Harper \& Brothers.

STANISH, Charles.

2003 Ancient Titicaca. The evolution of complex in Southern Peru and Northern Bolivia. University of California Press. 


\section{TANTALEÁN, Henry}

2005 Arqueología de la Formación del Estado. El caso de la cuenca norte del Titicaca. Lima, Auqi Ediciones.

TRIGGER, Bruce

1992 La Arqueología Histórico-Cultural. En Historia del Pensamiento Arqueológico. Editorial Crítica, Barcelona. Pp. 144-196.

VALCARCEL, Luis E.

2013 Luis Valcárcel: del Indigenismo cusqueño a la antropología peruana. Recopilado por José L. Reniqué y Luis F. Brugué. Tomo II. Petroperú SA, Fondo Editorial del Congreso del Perú e Instituto de Estudios Peruanos.

WILLEY, Gordon

1954 Wendell C. Bennett Memorial. American Antiquity, 19(3): 316.

UHLE, Max

1903 Pachacamac. University of Pennsylvania, Department of Archaeology, Philadelphia. 